\title{
Teste de susceptibilidade do percevejo, Cimex lectularius L. (Hemiptera, Cimicidae) ao DDT em Belo Horizonte, MG (Brasil)*
}

\author{
Susceptibility tests of the bed-bug Cimex lectularius L. (Hemiptera, Cimicidae) \\ to DDT in Belo Horizonte, MG (Brazil)
}

\begin{abstract}
Ronaldo L. Nagem**, Paul Williams**
NAGEM, R. L. \& WILLIAMS, P. Teste de susceptibilidade do percevejo Cimex lectularius L. (Hemiptera, Cimicidae) ao DDT em Belo Horizonte, MG (Brasil). Rev. Saúde públ., S. Paulo, 26: 125-8, 1992. O nível de susceptibilidade de percevejos adultos, Cimex lectularius, ao DDT, em Belo Horizonțe, Minas Gerais (Brasil), foi determinado durante o período de 1985 a 1986. Os resultados indicaram, para os insetos testados, que uma dose de $4,0 \%$ do inseticida não foi suficiente para matar $55 \%$ dos insetos. Os dados permitem concluir que existem colonias de percevejos resistentes ao DDT em Belo Horizonte.
\end{abstract}

Descritores: Percevejos. DDT. Resistência a inseticidas.

\section{Introdução}

Compostos clorados vêm sendo utilizados sistematicamente, em Minas Gerais, no controle e combate ao vetores da malária e da doença de Chagas, há pelo menos duas décadas. Mesmo não sendo objeto dessas campanhas, o percevejo de cama sofreu, indiretamente, os cfeitos que ficaram evidentes na redução de sua população na cilada região, embora $\mathrm{Nagem}^{10}$ (1985) tenha verificado a ocorrência de $C$. lectularius $\mathrm{cm}$ habitaçôcs humanas de Belo Horizonte e municípios vizinhos.

Pesquisas demonstram a resistência ou a susceptibilidade dos percevejos, aos compostos clorados, em diversas regiōes do mundo: Madden e col. ${ }^{7}$ (1945) com Cimex spp. c Mallis e Miller ${ }^{8}$ (1964) com C. lectularius, nos Estados Unidos; Chen e col. ${ }^{3}$ (1956) com $C$. hemipterus, em Taiwan; Brown ${ }^{2}$ (1958) com C. lectularius, $\mathrm{cm}$ Israel; Cristicescu c col. ${ }^{4}(1980)$ com C. lectularius, na Romenia c Mekuria e Tesfamarian' (1984) com C. lectularius, na Etiópia.

\footnotetext{
* Pesquisa financiada pelo Conselho Nacional de Desenvolvimento Científico e Tecnológico ( $\mathrm{CNl}_{\mathrm{q}}$ ). Processo $n^{2}$ 23072.062686/85-38. Pane da tese de doutorado "Cimex lectularius Linnacus da Região Metropolitana de Belo Horizonte, Minas Gerais: Observaçöes morfológicas e biológicas em laboratório", apresentada ao Instituto de Ciências Biológicas da UFMG, 1990.

** Departamento de Parasitologia - Instituto de Ciências Biológicas da UFMG - Belo Horizonte, MG - Brasil.
}

Separatas/Reprints: R. L. Nagem - Av. Antonio Carlos, 6627 C.P. 2486 - 31270 - 901 - Belo Horizonte, MG - Brasil
Gaaboub $^{6}$ (1971), utilizando o teste padrão da OMS, verificou, $\mathrm{cm}$ Alexandria (Egito), o nível de susceptibilidade de $C$. lectularius ao DDT e ao Dicldrin, no período de 1969-1970. Para o DDT a média da LC'50 (= concentração letal capaz de matar $50,0 \%$ dos insetos) foi de $2,00 \%$ com amplitude variada de 0,92 a $4,33 \%$; a média da LC'95 foi de $3,80 \%$ com amplitude variada de 1,75 a $8,23 \%$. Para o Dicldrin a média da LC'50 foi de $0,43 \%$ com amplitude variada de 0,14 a $1,31 \%$; a média da LC'95 foi de $0,82 \%$ com amplitude variada de 0,27 a $2,49 \%$. Tais resultados mostraram a presença de uma população contendo grupos com indivíduos susceptíveis, com indivíduos altamente resistentes ou com características intermediárias.

Uma maior percentagem de mortalidade de $C$. leciularius foi observada por Negromonte ${ }^{11}$ (1989), após 24 horas de exposição ao Malation cm comparação ao Baygon, Delumetrina, Lindane, Permetrina e DDT. Com 48 horas, após a exposição, o DDT c a Deltamctrina induziram uma maior mortalidade.

O presente trabalho teve como objelivo verificar a susceptibilidade de $C$. lectularius, provenientes da Regiño Metropolitana de Bclo Horizonte e mantidos $\mathrm{cm}$ laboratório sob condições ambientais, ao DDT, durante o período de 1985 a 1986.

\section{Material e Método}

A melodologia utilizada para a determinação do grau de susceptibilidade de cimicídeos ao DDT, foi a preconizada pela OMS ${ }^{12}$ (1970). 
Foram realizados três experimentos por prova. Em cada um foram utilizados 60 percevejos adultos retirados das colônias mantidas no laboralório do Setor de Entomologia, do Departamento de Parasitologia. Os percevejos adultos foram alimentados, um dia antes, e distribuídos em seis tubos de ensaio contendo, cada tubo, uma tira de papel impregnado com DDT nas concentraçōes: $0,25 \%, 0,50 \%$, $1,00 \%, 2,00 \%, 4,00 \%$ além do grupo controle. Os tubos foram fechados com gaze e deixados no laboratório por um periodo de 5 dias. Não foram usados artifícios nem técnicas que alterassem a umidade, a luminosidade ou a temperatura do laboratório.

Terminado o período de exposição ao inselicida, os percevejos mortos foram contados. Nos experimentos em que o frasco controle apresentou uma mortalidade superior a 5\%, as percentagens obtidas na prova foram corrigidas pela fórmula de Abbott, apresentada pela OMS $^{12}$ (1970). Os dados foram submetidos à análise de variância e de regressão linear mediante o pacote estatístico SAS (1985) ${ }^{13}$.

\section{Resultados}

A média da temperatura mínima $\mathrm{e}$ da máxima foi, durante os cinco dias de cada prova, de 22,0; 22,$0 ; 24,5^{\circ} \mathrm{C}$ e 22,$0 ; 24,0 ; 25,0^{\circ} \mathrm{C}$, respectivamente. Para a umidade a média da mínima e da máxima observada foi de 57,$0 ; 50,0 ; 73,5 \%$ c 59,0 ; 58,0; 76,0\%.

A Tabela apresenta os resultados obtidos das provas, com 3 experimentos cada, bem como a média da mortalidade total das três provas.

A Figura apresenta os pontos das curvas da susceptibilidade de $C$. lectularius ao DDT representadas em escala logarítmica da percentagem da mortalidade em rclação à concenuração do DDT. A linha traçada no gráfico corresponde ao logarítmo da média da percentagem de mortalidade.

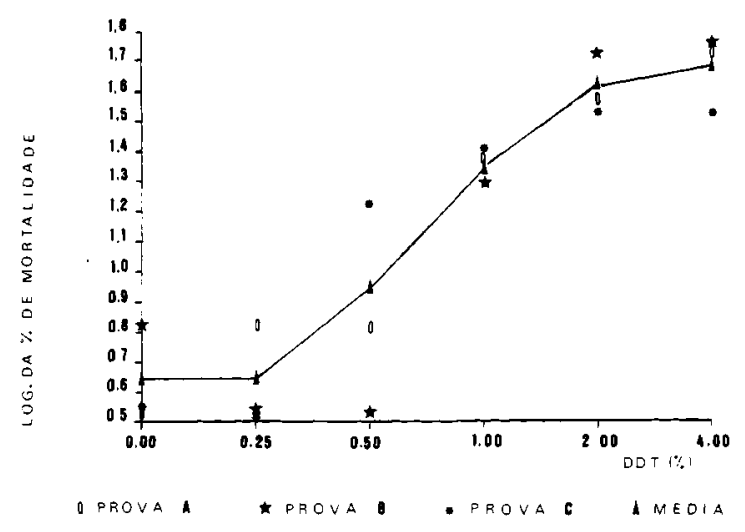

Flgura. Logaritmo da percentagem de mortalidade de $C$. lectularius exposto a diversas concentraçōes de DDT, durante 5 dias de provas, no periodo de 1985-86 em Belo Horizonte, MG

\section{Discussão}

Após uma análise de regressão linear, foi observado que as variáveis temperatura e umidade do ar não foram significativas, isto é, dentro dos limites propostos pela OMS ${ }^{12}$ (1970) essas variáveis não interferiram nos testes com o DDT.

Sendo a variável concentração associada à mortalidade dos percevejos, foi feita uma análise de variância nos níveis considerados $(0,00 \%$, $0,25 \%, 0,50 \%, 1,00 \%, 2,00 \%$ e $4,00 \%$ ). Foi observado que existe pelo menos um nível de inseticida que apresenta proporção de mortalidade diferente.

A análise estaústica, ao incluir os níveis de inseticidas $\mathrm{cm}$ grupos, explica $87,3 \%$ da mortalidade dos percevejos onde se verificou as suposições de variância constante e normalidade dos residuos.

Após a análise dos dados foi possivel concluir que as dosagens $0,00 \%, 0,25 \%$ e $0,50 \%$ devem ser consideradas num único grupo, por não apresentarem diferença significativa entre a proporção de

Tabela - Total de adultos de C. lectularius da Regiāo Metropolitana de Belo Horizonte, utilizados e mortos por prova A, B e $C$, expostos ao DDT durante 5 dias. $(M=$ Total de mortos. $M 1=$ Percentagem de mortos. $\mathrm{Ma}=$ mortalidade ajustada (Fórmula de Abbott)

\begin{tabular}{|c|c|c|c|c|c|c|c|c|c|c|c|c|c|}
\hline \multirow{2}{*}{$\begin{array}{l}\text { DDT } \\
(\%)\end{array}$} & \multirow[b]{2}{*}{ Total } & \multicolumn{3}{|c|}{$\begin{array}{l}\text { Prova A } \\
28 / 5 / 85\end{array}$} & \multicolumn{3}{|c|}{$\begin{array}{c}\text { Prova B } \\
8 / 8 / 85\end{array}$} & \multicolumn{3}{|c|}{$\begin{array}{c}\text { Prova C } \\
3 / 1 / 86\end{array}$} & \multicolumn{3}{|c|}{$\begin{array}{c}\text { Médias das } \\
\text { Provas }\end{array}$} \\
\hline & & $M$ & M1 & $\mathrm{Ma}$ & $M$ & M1 & $\mathrm{Ma}$ & $M$ & M1 & $\mathrm{Ma}$ & $M$ & M1 & $\mathrm{Ma}$ \\
\hline $\begin{array}{l}0,00 \\
0,25 \\
0,50 \\
1,00 \\
2,00 \\
4,00\end{array}$ & $\begin{array}{l}30 \\
30 \\
30 \\
30 \\
30 \\
30\end{array}$ & $\begin{array}{r}1 \\
2 \\
2 \\
7 \\
11 \\
16\end{array}$ & $\begin{array}{r}3,3 \\
6,7 \\
6,7 \\
23,3 \\
36,7 \\
53,3\end{array}$ & $\begin{array}{r}0,00 \\
3,45 \\
3,45 \\
20,69 \\
34,48 \\
51,72\end{array}$ & $\begin{array}{r}2 \\
1 \\
1 \\
6 \\
16 \\
17\end{array}$ & $\begin{array}{r}6,7 \\
3,3 \\
3,3 \\
20,0 \\
53,3 \\
56,7\end{array}$ & $\begin{array}{r}0,00 \\
-3,57 \\
-3,57 \\
14,29 \\
50,00 \\
53,57\end{array}$ & $\begin{array}{r}1 \\
1 \\
5 \\
7 \\
10 \\
10\end{array}$ & $\begin{array}{r}3,3 \\
3,3 \\
16,7 \\
23,3 \\
33,3 \\
33,3\end{array}$ & $\begin{array}{r}0,00 \\
0,00 \\
13,79 \\
20,69 \\
31,03 \\
31,03\end{array}$ & $\begin{array}{r}1,3 \\
1,3 \\
2,7 \\
6,7 \\
12,3 \\
14,3\end{array}$ & $\begin{array}{r}4,4 \\
4,4 \\
8,9 \\
22,2 \\
41,1 \\
47,8\end{array}$ & $\begin{array}{r}0,00 \\
0,00 \\
4,65 \\
18,60 \\
38,37 \\
45,35\end{array}$ \\
\hline
\end{tabular}


percevejos mortos. A mortalidade média de cada grupo foi: $1^{9}$ grupo - $5,9 \%(0,059) ; 2^{9}$ grupo $22,2 \%(0,222)$ e o $3^{\circ}$ grupo $-44,4 \%$.

Pela Tabela a mortalidade dos percevcjos não ultrapassa a 57,0\% mesmo com a dose máxima de $4,0 \%$, (56,7\% na prova $B$ e $33,3 \%$ na C) o que indica a presença de indivíduos pouco susceplíveis ao DDT com acentuada resistência fisiológica.

A Tabela mostra, tambćm, os resultados corrigidos pela fórmula de Abbott, confirmando que $\mathrm{cm}$ cada um dos três experimentos, realizados $\mathrm{cm}$ diferentes épocas do ano com diferentes geraçōes de $C$. lectularius, os cimicídeos originados da Região Metropolitana de Belo Horizontc, mostraram-se pouco susceptíveis ao DDT.

Para Forattini ${ }^{5}(1962)$ a $\mathrm{DL}_{50}$ pode ser calculada na prova que apresenta uma mortalidade acima de $99 \%$, nos demais casos o cálculo pode dar uma idéia errada, pois a população não é normal e homogênea. Na Figura (logarítmo da média da percentagem de mortalidade nas provas $\mathrm{A}, \mathrm{B}, \mathrm{e}$ C) pode-se observar a presença de uma baixa susceptibilidade ao inseticida testado. Convém salientar que nem nos testes preliminares foi possível obter uma mortalidade próxima de $90 \%$ para o percevejo de cama.

Uma mortalidade inferior a $50 \%$ para C. lectularius, proveniente da mesma localidade, porćm de época distinta, foi constatada por Negromonte ${ }^{11}$ (1989), usando a Permetrina (Pircı́óide).

Os presentes resultados foram difcrentes, para C. lectularius, dos apresentados por Madden e col. ${ }^{7}$ (1945), quando obliveram uma mortalidade de 100,0\% para as duas espécies de Cimex usando DDT em pó, a 5,0\% depois de $24 \mathrm{~h}$ de aplicação. Essa percentagem caiu para $87,0 \%$ quando cle usou DDT em óleo mincral. Da mesma forma, diferem dos resultados de Bishopp ${ }^{1}$ (1946) quando considerava o DDT a $5,0 \% \mathrm{~cm}$ querosene como o melhor tratamento a ser utilizado no combate aos percevejos.

A alta taxa de resistência, demonstrada após cinco dias de exposição ao DDT, para os percevejos, pode ser decorrente do uso indiscriminado do inseticida em aerosol utilizado pelos moradores.

É importante considerar que os moradores são, geralmente, procedentes de regiōes rurais onde essas campanhas são mais intensas. Ao migrarem para os centros urbanos, esses moradores poderiam trazer, em seus pertences, o percevcjo já resistente ao inseticida. Estudos futuros poderiam scr realizados em regiozes expostas aos inselicidas utilizados em campanhas, com o objetivo de comparar e estender as observações para todo o País.

Além dessas observações convém ressaltar que, normalmente, as favelas ou habitaçõcs da periferia da Região Metropolitana de Belo Horizonte (de onde foram colctados os percevejos para o estabelecimento de colônias cm laboratório) não são alvos constantes de campanhas de erradicação da malária e doença de Chagas.

Observaçōes de Nagem, não publicadas*, indicam que os moradores das residências visitadas e infestadas por percevejos, descrentes já quanto ao uso de qualquer inseticida no combate aos percevejos, só vêcm uma solução no combate aos mesmos. Esta consistc em queimar todos os objetos e pertences da casa e em alguns casos até a própria moradia.

Os presentes resultados permitem incorporar 0 Brasil, quando os testes com $4 \%$ de DDT indicaram uma mortalidade, de $C$. lectularius, de menos de $60 \%$ dos percevejos testados, à lista dos países com casos de percevejos resistentes ao DDT.

\section{Agradecimentos}

À Dra. Rotraut Anna G. Consoli, do Centro de Pesquisas Renné Rachou - FIOCRUZ - Belo Horizonte, pelo fornecimento do papel impregnado com DDT.

NAGEM, R.L. \& WILLIAMS, P. [Susceptibility tests of the bed-bug Cimex lectularius L., (Hemiptera, Cimicidac) 10 DDT in Belo Horizonte, MG. (Brazil)]. Rev. Saúde públ., S. Paulo, 26: 125-8, 1992. Susceptibility levels for the adult bed-bug, Cimex lectularius, in Belo Horizonte, Minas Gerais - Brazil, to DDT, were determined during the period 1985 to 1986 . The test results showed that a $4 \%$ dosage of DDT is not enough to kill 55\% of the insects. The data are sufficient to show that there exists bed-bug resistance to DDT in Belo Horizonte.

Keywords: Bed-bugs. DDT. Insecticide resistance.

* Nagem, R. L. Comunicação pessoal, 1982.

\section{Referências Bibliográficas}

1. BISIIOPP, F. C. Present position of DDT in the control of inscas of medical importance. Amer. J. publ. Wlth, 36: 593-600, 1946.

2. BROWN, A. W. A. The insecticide resistance problem: a review of developments in 1956 and 1957. Bull. Wld lllth Org., 18: 309-21, 1958.

3. CIIIEN, II.-II; TENG, P.-T; PLETSCII, D. J. DDT resistant bed-bugs (Cimex hemiplerus Fabr.) found in an anny camp in Southem Taiwan, China. J. Formosan med. Ass., 55: 143-8, 1956.

4. CRISITCLSCU, A.; GIURCÃ, I.; DURBACÃ, S. The surveillance or the resistance to insecticides in the Cimex leclularius (Heteroptera, Cimicidac) species. Arch. roum. Path exp. Microbiol, 39: 171-7, 1980. 
5. FORATTINI, O. P. Determinação da resistência fisiológica ou da susceptibilidade aos inseticidas (Técnica da Organização Mundjal de Saúde, OMS, 1959). In: Foratini, O. P. Entomologia médica. São Paulo, Faculdade de Higiene e Saúde Pública, 1962. v. 1, p. 625-36.

6. GAABOUB, I. A. Present state of DDT and dicldrin resistance in the bed-bug, Cimex lectularius in Alexandria District, United Arab Republic. Z. Angeusw. Ent., 68: 176-80, 1971.

7. MADDEN, A. H.; LINDQUIST, A. W.; KNIPLING, E. F. DDT and other insecticides as residual-type treatments to kill bed-bugs. J. econ. Ent., 38: 265-71, 1945.

8. MALLIS, A.\& MILLER, A.C. Prolonged resistance in the house fly and bed-bug J. econ. Ent., 57: 608-9, 1964.

9. MEKURIA, Y. \& TESFAMARIAM, T. The susceptibility of some insect populations in Addis Ababa, Echiopia to DDT and Malathion. East Afr. med.J., 61:618-23, 1984.

10. NAGEM, R. L. Ocorrência de Cimex lectularius L., 1758 (Hemiptera, Cimicidae) em algumas habitações huma- nas de Belo Horizonte e municípios vizinhos. Rev. bras. Ent., 29: 217-20, 1985.

11. NEGROMONTE, M. R. S. A infestaçāo pelo Cimex em uma comunidade piloto de Belo Hlorizonte, MG (Bairro Gorduras) prevalência, perspectivas e eficácia de inseticidas comerciais. Belo Horizonte, 1989. [Dissertação de Mestrado - Departamento de Parasitologia da UFMG].

12. ORGANTZACION MUNDIAL DE LA SALUD. Comite de Expertos en Insecticidas, Ginebra, 1968. Resistencia a los insecticidas y lucha contra los vectores; $17^{\mathrm{B}}$ informe. Ginebra, 1970. (Série de Informes Técnicos, 443).

13. SAS INSTITUTE DNC. Sas users guide: basics; version 5 Edition. Cary, N.C., 1985.

Recebido para publicaçâo em 31/7/1991 Reapresentado em 22/11/1991 Aprovado para publicaçāo em 29/11/1991 